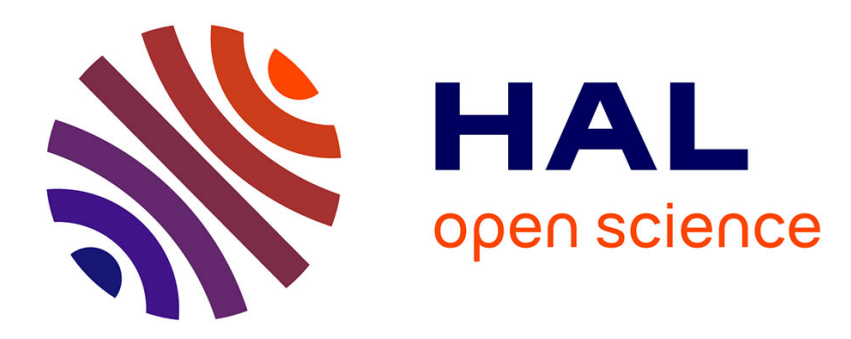

\title{
A dynamical theory of elastic dielectrics
}

\author{
R. A. Toupin
}

\section{- To cite this version:}

R. A. Toupin. A dynamical theory of elastic dielectrics. International Journal of Engineering Science, 1963, 1 (1), pp.101-126. hal-00852604

\section{HAL Id: hal-00852604 https://hal.science/hal-00852604}

Submitted on 26 Aug 2013

HAL is a multi-disciplinary open access archive for the deposit and dissemination of scientific research documents, whether they are published or not. The documents may come from teaching and research institutions in France or abroad, or from public or private research centers.
L'archive ouverte pluridisciplinaire HAL, est destinée au dépôt et à la diffusion de documents scientifiques de niveau recherche, publiés ou non, émanant des établissements d'enseignement et de recherche français ou étrangers, des laboratoires publics ou privés. 


\title{
A DYNAMICAL THEORY OF ELASTIC DIELECTRICS*
}

\author{
R. A. ToupIN \\ International Business Machines Corporation Thomas J. Watson Research Center, \\ Yorktown Heights, N.Y.
}

\begin{abstract}
A theory of the electromagnetic field in moving, finitely deformed elastic dielectrics is developed. The equations for a weak field superimposed on a strong field and an arbitrary state of finite deformation are deduced. It is shown how certain electro- and magneto-optical effects and the retardation of a sound wave by a strong magnetic field in a dielectric can be explained and correlated.
\end{abstract}

\section{INTRODUCTION}

IN A PREVIOUS paper called 'The Elastic Dielectric' [1] we considered the finite deformation of and the electrostatic field in perfectly elastic dielectrics. In this paper we extend our results so as to include the effects of motion and deformation on the electromagnetic field in perfectly elastic dielectrics.

Although Maxwell's equations for the electromagnetic field in vacuum are linear so that the sum of two solutions is again a solution, in dielectric media, they are not. The response of a material to weak oscillatory electromagnetic fields (i.e. the optical properties of a material) can be altered by placing it in a strong electric or magnetic field. The electroand magneto-optical effects which are observed are direct evidence that the equations for the electromagnetic field in a material medium are essentially non-linear.

The general non-linear theory of elastic dielectrics set forth in the following pages is designed to present a unified mathematical theory of the piezoelectric, photoelastic, and electro- and magneto-optical properties of elastic dielectrics. We have not considered thermal effects, and no attempt has been made to treat magnetization. In our opinion, all presently known treatments of magnetization in moving media are defective. A careful treatment of magnetized dielectric media in motion offers an interesting direction for future development of the theory of the electromagnetic field in deformable elastic media.

Certainly, no pronounced or obvious property of ordinary elastic dielectrics has escaped the notice of both theorists and experimentalists. On the other hand, the equations of the theory developed here reveal more than one subtle phenomenon which might be extremely difficult to detect in a real material and without engineering application, but which might serve to support or overturn the theory.

\section{THE EQUATIONS OF BALANCE}

Underlying all purely mechanical theories of elastic bodies and ordinary fluids are four fundamental principles of conservation. These are (1) conservation of mass, (2) conservation of linear momentum, (3) conservation of angular momentum, and (4) conservation of energy. The following integral equations of balance express these basic principles of mechanics in mathematical form sufficiently general for our purposes.

* The major portion of this paper was written while the author was a member of the Applied Mathematics Staff of the U.S. Naval Research Laboratory, Washington 25, D.C. 
(1) Mass

$$
\frac{\mathrm{d}}{\mathrm{d} t} \int_{v} \rho \mathrm{d} v=0
$$

(2) Linear Momentum

$$
\frac{\mathrm{d}}{\mathrm{d} t} \int_{v} p_{i} \mathrm{~d} v=\oint_{s} t_{i}^{j} \mathrm{~d} a_{j}+\int_{v} f_{i} \mathrm{~d} v
$$

(3) Angular Momentum

$$
\frac{\mathrm{d}}{\mathrm{d} t} \int_{v}\left(s_{k i}+x_{[k} p_{i]}\right) \mathrm{d} v=\oint_{s}\left(m_{k i}{ }^{i}+x_{[k} t_{i]}{ }^{j}\right) \mathrm{d} a_{j}+\int_{v}\left(l_{k i}+x_{[k} f_{i]}\right) \mathrm{d} v .
$$

(4) Energy

$$
\begin{aligned}
\frac{\mathrm{d}}{\mathrm{d} t} \int_{s} U \mathrm{~d} v=\oint_{s}\left(t_{i}{ }^{j} \dot{x}^{i}-m^{i k j} \dot{x}_{[i, k]}\right) \mathrm{d} a_{j}+\int_{v}\left(f_{i} \dot{x}^{i}-l^{i j} \dot{x}_{[i, j]}\right) \mathrm{d} v & \\
& +\oint_{s} h^{j} \mathrm{~d} a_{j}+\int_{v} q \mathrm{~d} v .
\end{aligned}
$$

For simplicity, in writing the second and third of the foregoing integral equations of balance which contain vector valued integrals we have assumed that all quantities are expressed in a common rectilinear frame of reference. The region of integration $v$ is a moving region, in general, which contains the same set of material points at each instant $t$ and $s$ is the complete boundary of $v$. The equations of balance hold for arbitrary regions $v$. The quantities which occur in them are named as follows:

$$
\begin{aligned}
\rho & =\text { mass density, } & m_{i j} & =\text { couple-stress } \\
p_{i} & =\text { momentum density, } & l_{i j} & =\text { extrinsic body couple }, \\
t_{i}^{j} & =\text { stress tensor }, & U & =\text { energy density }, \\
f_{i} & =\text { extrinsic body force, } & h^{i} & =\text { extra flux of energy }, \\
s_{j i} & =\text { spin density, } & q & =\text { supply of energy. }
\end{aligned}
$$

Each of these equations of balance has the typical structure

$$
\frac{\mathrm{d}}{\mathrm{d} t} \int_{v} Q \mathrm{~d} v=\oint_{s} F^{j} \mathrm{~d} a_{j}+\int_{v} S \mathrm{~d} v
$$

where $Q$ is the density of the quantity in balance, $F^{j}$ is its flux relative to the material, and $S$ is its supply. From (2.1) we see that the flux and supply of mass are always zero. From (2.2) we see that the stress tensor is the flux of momentum and the extrinsic body force is the supply of linear momentum. If the material interacts with a gravitational field, the supply of linear momentum is given by $\rho g_{i}$, where $g_{i}$ is the gravitational field strength. This is the case usually considered in elasticity theory. Such a body force represents the rate at which the material gains or loses linear momentum from or to the gravitational field. From the equation of balance of angular momentum (2.3) we see that the density of angular momentum consists in a sum of two terms, $s_{i j}$ and $x_{[i} p_{j]}$. The former, the spin density, is the excess in the density of angular momentum over the moment of linear momentum about a fixed point which we have taken as the origin of the co-ordinate system. The flux of angular 
momentum consists also in the sum of two contributions, $m_{k i}{ }^{j}$, the couple-stress, and $x_{[k} t_{i]}{ }^{j}$, the moment of the flux of linear momentum about the origin. The supply of spin is the sum of two parts with similar properties. Turning now to the energy equation, it is possible here also to discuss the intuitive meaning of each term in the flux and supply of energy. The physical interpretation is promoted by calling $t_{i}^{j} \dot{x}^{i}$, the rate of working of the stress; $-m^{i k} \dot{x}_{[i, k]}$, the rate of working of the couple-stress; $f_{i} \dot{x}^{i}$, the rate of working of the extrinsic body force; and $l^{i j} \dot{x}_{[i, j]}$, the rate of working of the body couples.

Theories of continuous media which assume that the spin density, the couple-stress, and the extrinsic body couples all vanish are called apolar as opposed to polar theories in which one or more of these quantities fails to vanish. The equations of balance of mass, linear momentum, angular momentum, and energy form merely the framework upon which a theory of motion of a continuous medium is built. The equations of balance must be supplemented by a system of constitutive relations expressing the various fluxes, densities, and supplies which appear in them in terms of an independent set of kinematical and other variables describing the state of motion, the electromagnetic condition, or thermodynamic state of the medium. In the theory of elastic dielectrics to be constructed here, they are supplemented also by the basic laws of electromagnetism. The equations of balance are common to all mechanical theories. It is the details of the constitutive relations which distinguish one continuum theory from another. The constitutive relations may be as simple or as complex as one desires depending on his aims. Our objective in the following pages is to set up the basic constitutive relations of a relatively simple theory of elastic dielectrics. The constitutive relations will be patterned after those of the classical theory of finite elastic deformations where the electromagnetic field does not appear.

\section{THE CONSTITUTIVE RELATIONS OF ORDINARY ELASTICITY THEORY}

As preparation for the more complex problem of dielectrics, let us review very briefly how onc may arrive at the equations of finite elastic deformations of ordinary elastic bodies. The pattern of reasoning in the case of dielectrics will be similar to the following.

Let the motion of the medium be described by a mapping

$$
x^{i}=\tilde{x}^{i}\left(X^{1}, X^{2}, X^{3}, t\right)
$$

where $t$ is the time; $X^{A}, A=1,2,3$ are the co-ordinates of a material point $\mathbf{P}$ in some reference configuration $C_{0}$; and $x^{i}, i=1,2,3$ are the co-ordinates at time $t$ of the material point $\mathbf{P}$. The mapping (3.1) is assumed to be one-to-one and twice continuously differentiable throughout a domain $B=\{\mathbf{X}\}$ called the body. Thus $(\mathbf{x} / \mathbf{X})=\operatorname{det}\left\|x^{i},{ }_{A}\right\|$, where $x^{i},{ }_{A} \equiv$ $\partial \tilde{x}^{i} / \partial X^{A}$, is different from zero and (3.1) has a unique inverse $X^{A}=\tilde{X}^{A}\left(x^{1}, x^{2}, x^{3}, t\right)$ with Jacobian matrix $X^{A}{ }_{, i} \equiv \partial \tilde{X}^{A} / \partial x^{i}$. The velocity of the medium is defined by

$$
\dot{x}^{i} \equiv \frac{\partial \dot{x}^{i}}{\partial t}
$$

and the acceleration by

$$
\ddot{x}^{i} \equiv \frac{\partial^{2} \ddot{x}^{i}}{\partial t^{2}}
$$

In all that follows, as in (3.2) and (3.3), we use a superposed dot to denote differentiation with respect to $t$ holding the material co-ordinates $X^{A}$ fixed. The quantities $x^{i},{ }_{A}$ are the components of the deformation gradient $\mathbf{F}$. 
The classical theory of finite elastic deformations is an apolar theory based upon the following list of further assumptions:

$$
\begin{aligned}
& \begin{array}{l}
p_{i}=\rho \dot{x}_{i}, \quad t_{i}{ }^{j}=\tilde{t}_{i}^{j}(\mathbf{F}), \quad f_{i}=\rho g_{i}, \\
U=\rho(\Sigma+\kappa), \quad \text { where } \quad k \equiv \frac{1}{2} \dot{x}^{2} \quad \text { (kinetic energy per unit mass) } \\
\Sigma \equiv \tilde{\Sigma}(\mathbf{F}) \text { (internal energy per unit mass) }
\end{array} \\
& h^{i}=q=0 \quad \text { (neglect of thermal effects) }
\end{aligned}
$$

The elastic qualities of the material are embodied in the two basic assumptions in (3.4) where it is postulated that the stress $t_{i}^{j}$ and the internal energy $\Sigma$ are functions of the deformation gradient F. Thermoelasticity theory could be incorporated in the above scheme by allowing the internal energy and the stress to depend also upon the entropy density or temperature and by replacing the assumption $h^{i}=0$ by a suitable law of heat conduction. The theory of elastic dielectrics to be developed may also be extended to cover thermal phenomena in a similar way.

Once it is assumed that the momentum and energy densities have the form given in (3.4) the field equations which follow from the integral equations of balance assume the following form:

$$
\begin{gathered}
\rho \ddot{x}^{i}=t^{i j}, j+\rho g_{i}, \\
t^{[i j]}=0, \\
\rho \dot{\Sigma}=t^{i j} \dot{x}_{i, j}, \\
\frac{\partial \rho}{\partial t}+\left(\rho \dot{x}^{i}\right)_{, i}=0 .
\end{gathered}
$$

Equation (3.8) is the familiar continuity equation. Its general solution is

$$
\rho=|(\mathbf{x} / \mathbf{X})|^{-1} \rho_{\circ}(\mathbf{X}),
$$

where $\rho_{\mathrm{o}}(\mathbf{X})$ is the density of mass in the reference state. Using (3.9) we may eliminate $\rho$ from the system (3.5)-(3.7). This system of equations then consists in a total of $3+3+1$ $=7$ independent equations in the three unknown quantities $\tilde{x}^{i}\left(X^{1}, X^{2}, X^{3}, t\right)$. Thus it appears that the system is overdetermined. To render the number of equations and unknowns equal, it is sufficient to require that equations (3.6) and (3.7) be satisfied identically by every motion. For (3.7) to be satisfied identically it is necessary and sufficient that the stress functions $\mathcal{Z}_{i}^{j}(\mathbf{F})$ and the energy function $\tilde{\Sigma}(\mathbf{F})$ be related according to

$$
\tilde{t}_{i}^{j}=\rho \frac{\partial \tilde{\Sigma}}{\partial x^{i}, A} x^{j}, A
$$

Equation (3.6) then requires that the internal energy function satisfy the three independent relations

$$
\frac{\partial \tilde{\Sigma}}{\partial x_{[i, A}} x^{j]}, A=0
$$


This equation will be satisfied by every motion if and only if the internal energy is invariant under all rigid motions (cf. $[1, \S 10]$ ). It must then be expressible as a function of the measures $E_{A B}$ of finite strain given by

The stress is then given by

$$
\begin{aligned}
& E_{A B} \equiv \frac{1}{2}\left(C_{A B}-g_{A B}\right), \\
& C_{A B} \equiv g_{i j} x_{, A} x_{, B}^{j} .
\end{aligned}
$$

$$
t^{i j}=\rho \frac{\partial \widetilde{\Sigma}}{\partial E_{A B}} x^{i}{ }_{, A} x^{i},
$$

where

$$
\Sigma=\tilde{\Sigma}(\mathbf{E}) .
$$

With the internal energy and stress given as in (3.13) and (3.14), equations (3.6) and (3.7) are satisfied identically by every motion. One is left then with the three equations of motion (3.5) for determining the three functions $\tilde{x}^{i}$ subject to suitable boundary and initial conditions. Of course, not every function $\tilde{\tilde{\Sigma}}(\mathbf{E})$ can possibly represent the internal energy of a real elastic material. Certain restrictions upon the form of this function are satisfied by every known material, but this aspect of the theory of elasticity and of its generalization to dielectrics to follow will not be discussed in this paper.

We shall arrive at the constitutive relations of an elastic dielectric by a route which is a slight modification of the foregoing sequence of ideas. The dielectric theory will be so constructed that, upon neglect of all electromagnetic effects, its equations will reduce to those just given. But first we give an independent review of the electromagnetic equations for moving and deforming dielectrics which will complement the equations of balance of mass, momentum, angular momentum, and energy already introduced and form the remaining part of the foundation of the theory of elastic dielectrics.

\section{THE ELECTROMAGNETIC FIELD IN DIELECTRICS}

The Maxwell-Lorentz theory of the electromagnetic field in polarizable, non-conducting, non-magnetic, deformable media is based upon the following system of equations relating the electric field $\mathbf{E}$, the magnetic flux density $\mathbf{B}$, and the polarization $\mathbf{P}$.

$$
\begin{gathered}
\frac{\mathrm{d}}{\mathrm{d} t} \int_{s} \mathbf{B} \cdot \mathrm{d} \mathbf{a}+\oint_{c} \mathbf{E} \cdot \mathrm{d} \mathbf{x}=0, \quad \oint_{s} \mathbf{B} \cdot \mathrm{d} \mathbf{a}=0, \\
\frac{1}{\mu_{\circ}} \oint_{c} \mathbf{B} \cdot \mathrm{d} \mathbf{x}-\frac{\mathrm{d}}{\mathrm{d} t} \int_{s} \epsilon_{\circ} \mathbf{E} \cdot \mathrm{d} \mathbf{a}=\int_{s}^{*} \mathbf{P} \cdot \mathrm{d} \mathbf{a}-\int_{s}(\operatorname{div} \mathbf{P}) \dot{\mathbf{x}} \cdot \mathrm{d} \mathbf{a} \\
\oint_{\epsilon_{\circ} \mathbf{E} \cdot \mathrm{d} \mathbf{a}}=-\oint_{\mathbf{P} \cdot \mathrm{d} \mathbf{a} .}
\end{gathered}
$$

These equations must hold for every stationary circuit $c$ or surface $s$ in an inertial frame. In (4.1) and (4.2), $c$ is the complete boundary of the surface $s$. Equations (4.1) are the mathematical expression of Faraday's law of magnetic induction. Equations (4.2) and (4.3) express the fundamental relation between the electromagnetic field and the distribution of charge and current in dielectric media. In non-conducting dielectric media which are also 
non-magnetic, the current density $\mathbf{J}$ consists solely of the polarization current, $\mathbf{J}=$ $\mathbf{P}-(\operatorname{div} \mathbf{P}) \dot{\mathbf{x}}$, where $\mathbf{P}_{\mathbf{P}}^{*}$ is the convected time derivative of the polarization density given in terms of $\mathbf{P}$ and the velocity of the medium by the formula

$$
\stackrel{*}{P}^{i}=\frac{\partial P^{i}}{\partial t}+\dot{x}^{j} P_{, j}^{i}-\dot{x}_{, j}^{i} P^{j}+\dot{x}_{, j}^{j} P^{i}
$$

which is equivalent to

$$
\stackrel{*}{\mathbf{P}}=\frac{\partial \mathbf{P}}{\partial t}+(\operatorname{div} \mathbf{P}) \dot{\mathbf{x}}+\operatorname{curl}(\mathbf{P} \times \dot{\mathbf{x}}) .
$$

The integral on the right-hand side of (4.3) represents the total bound charge in the interior of the closed surface $s$. The fundamental constants $\mu_{0}$ and $\epsilon_{0}$ in (4.2) and (4.3) are related to the speed of light in vacuum by $\mu_{\circ} \epsilon_{\circ}=c^{-2}$.

For our present purposes, we lay down equations (4.1)-(4.4) as postulates. A derivation and discussion of these equations beginning from first principles has been given in [2, Chap. F]. An elementary derivation is given by Whittaker [5, pp. 396-402].

If one introduces the definitions

$$
\mathbf{H} \equiv \mu_{\circ}^{-1} \mathbf{B}+\dot{\mathbf{x}} \times \mathbf{P}, \quad \mathbf{D} \equiv \epsilon_{\circ} \mathbf{E}+\mathbf{P}
$$

then (4.2) and (4.3) may be put in the form

$$
\oint_{c} \mathbf{H} \cdot \mathrm{d} \mathbf{x}-\frac{\mathrm{d}}{\mathrm{d} t} \int \mathbf{D} \cdot \mathrm{d} \mathbf{a}=0, \quad \oint \mathbf{D} \cdot \mathrm{d} \mathbf{a}=0 .
$$

If $\mathbf{B}$ is transformed as an axial vector density of weight $1, \mathbf{E}$ as an absolute polar vector of weight zero, and $\mathbf{P}$ as a polar density of weight 1 , the field equations which follow from the integral equations (4.1)-(4.3) may be expressed in the following form which is invariant under general time independent transformations of the spatial co-ordinates.

$$
\begin{array}{ll}
\frac{\partial B^{i}}{\partial t}+e^{i j k} \partial_{j} E_{k}=0, & \partial_{i} B^{i}=0, \\
e^{i j k} \partial_{j} H_{k}-\frac{\partial D^{i}}{\partial t}=0, & \partial_{i} D^{i}=0,
\end{array}
$$

where

$$
\begin{aligned}
H_{k} & =g_{k m} B^{i} /\left(\mu_{\circ} \sqrt{g}\right)+e_{k m n} \dot{x}^{m} P^{n}, \\
D^{i} & =\epsilon_{\circ} \sqrt{ }(g) g^{i j} E_{j}+P^{i},
\end{aligned}
$$

and $e_{i j k}=e_{[i j k]}$ is the completely antisymmetric axial tensor density for which $e_{123}=1$. This form of the equations is particularly convenient for calculations in curvilinear coordinate systems since only ordinary partial derivatives appear. The transformations laws we have chosen for $\mathbf{E}, \mathbf{B}$, and $\mathbf{P}$ agree with the traditional ones under the restricted group of orthogonal transformations, including inversions of a spatial axis. We should caution that some authors prefer to transform all vector quantities as tensors of weight zero. Thus, in a curvilinear system of co-ordinates, some authors write the system of equations (4.8) in terms of $\overline{\mathbf{B}}=\mathbf{B} / \sqrt{ } g, \mathbf{E}$, and $\overline{\mathbf{P}} / \sqrt{ } g$. These different schemes for transforming the basic field quantities are simply a matter of bookkeeping and one may consistently use any scheme one 
prefers. The reader who wishes to view all equations as written in rectangular Cartesian co-ordinate systems only, need not be concerned with these details of the tensor calculus.

We insert here the caution: The Lorentz theory of the electromagnetic field in dielectrics which we use here is not relativistic (i.e. Lorentz invariant). On the other hand, for non-magnetizable materials, the Lorentz theory agrees with the relativistic theory of moving media developed by Minkowski [3], and by Einstein and Laub [4], to within terms of order $v^{2} / c^{2}$, where $v$ is the speed of the medium relative to the frame of reference. The matter is discussed by Einstein and Laub in the paper cited. For correct interpretation of the Lorentz theory, it is necessary for the inertial frame of reference relative to which the quantities in (4.8)-(4.9) are measured to be stationary with respect to the aether. This simply reflects the fact that this system of equations is neither Lorentz invariant nor Galilean invariant. The equations are not form invariant under transformations to moving systems of reference. But for a description of the electromagnetic field in moving media where the speed of the medium is everywhere small compared with the speed of light in vacuum, the Lorentz equations are satisfactory.

The system of field equations (4.8)-(4.9) is underdetermined. There appears in them a total of twelve unknowns, $B^{i}, E_{i}, p^{i}$, and $\dot{x}^{i}$. Even if the motion be regarded as prescribed, it is necessary to complement this system of equations with three further relations amongst the unknowns. The simplest example of such an additional relation is afforded by the rigid, linear, polarizable medium for which one assumes that

$$
\mathbf{P}=\epsilon \circ \chi(\mathbf{E}+\dot{\mathbf{x}} \times \mathbf{B}) .
$$

Voigt [6] considered generalizations of this simple relation having the following general form

$$
\begin{gathered}
\mathbf{P}_{\alpha}+\mathbf{a}_{\alpha} \cdot \frac{\partial^{2} \mathbf{P}_{\alpha}}{\partial t^{2}}+\mathbf{b}_{\alpha} \cdot \frac{\partial \mathbf{P}_{\alpha}}{\partial t}+\mathbf{c}_{\alpha} \cdot\left(\frac{\partial \mathbf{P}_{\alpha}}{\partial t} \times \mathbf{B}\right)={ }_{\circ} \epsilon \boldsymbol{\chi}_{\alpha} \cdot \mathbf{E} \\
\mathbf{P}=\sum_{\alpha} \mathbf{P}_{\alpha} .
\end{gathered}
$$

Voigt's theory based on a constitutive relation of the form (4.11) applies only to rigid and stationary media. In these equations $\mathbf{P}_{\alpha}$ is the contribution to the total polarization of the molecular species $\alpha$. The coefficients $\mathbf{a}_{\alpha}$ are a measure of the importance of inertia in the equations of motion for the species $\alpha$; the $\mathbf{b}_{\alpha}$ measure a type of linear resistive and dissipative force; and the $\mathbf{c}_{\alpha}$ give rise to the Faraday rotation and optical activity of the medium. In terms of such a system of differential constitutive relations, Voigt was able to explain the absorption and dispersion of light by a stationary medium. We cite this example of a constitutive relation simply to illustrate the variety of different theories embraced by the basic equations (4.1)-(4.3). The theory of perfectly elastic dielectrics developed in the following sections is valid for moving and deforming media; hence, in one sense, it is more general than Voigt's. On the other hand, it is less general since it neglects all dissipative phenomena.

\section{THE EQUATIONS OF MOTION AND CONSTITUTIVE RELATIONS FOR PERFECTLY ELASTIC DIELECTRICS}

Consider the equations of balance of mass, linear momentum, angular momentum, and energy (2.1)-(2.4). We base this theory of perfectly clastic dielectrics on the following 
special assumptions which replace the constitutive assumptions (3.4) of ordinary elasticity theory.

$$
\begin{aligned}
& \left.p_{i}=\rho \dot{x}^{i}, \quad t_{i}^{j}=\tilde{t}_{i}^{j}(\mathbf{F}, \mathbf{P}), \quad f_{i}=-\operatorname{div} \mathbf{P} \mathscr{E}_{i}+\stackrel{*}{(\mathbf{P}} \times \mathbf{B}\right)_{i} \\
& s_{i j}=m_{i j k}=l_{i j}=0, \\
& U=\rho(\Sigma+\kappa), \quad \Sigma=\hat{\Sigma}(\mathbf{F}, \mathbf{P}), \quad \kappa=\frac{1}{2} \dot{x}^{2} \\
& h^{i}=0, \quad q=\stackrel{*}{\mathbf{P}} \cdot \mathscr{E} .
\end{aligned}
$$

In these equations we have introduced the notation $\mathscr{E}=\mathbf{E}+\dot{\mathbf{x}} \times \mathbf{B}$ for the electromotive intensity. Assumption (5.1) $)_{3}$ is motivated as follows. In Lorentz' theory, the quantity

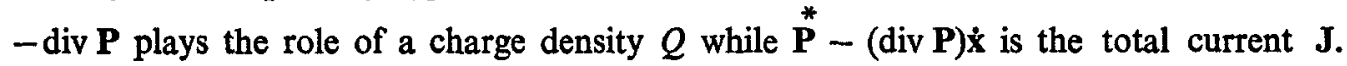
Thus the extrinsic body force (5.1) $)_{3}$ is the Lorentz force $Q \mathbf{E}+\mathbf{J} \times \mathbf{B}$. It represents the rate at which the material gains or loses linear momentum from or to the electromagnetic field. The expression we have postulated for the supply of energy $q$ is the usual expression $\mathbf{J} \cdot \mathbf{E}$ for the Joule power less the rate of working of the extrinsic body force. That is, we have $\mathbf{J} \cdot \mathbf{E}=(\stackrel{*}{\mathbf{P}}-\operatorname{div} \mathbf{P} \dot{\mathbf{x}}) \cdot \mathbf{E}=\stackrel{*}{\mathbf{P}} \cdot \mathscr{E}+\mathbf{f} \cdot \dot{\mathbf{x}}$, where $\mathbf{f}$ is given by $(5.1)_{3}$. Thus $\mathbf{J} \cdot \mathbf{E}$ represents the rate at which the material gains kinetic and internal energy from the electromagnetic field. On substituting the expressions (5.1) into the general equations of balance (2.1)-(2.4) and applying the divergence theorem in the usual way, we arrive at the field equations

$$
\begin{gathered}
\rho \ddot{x}^{i}=t^{i j}-(\operatorname{div} \mathbf{P}) \mathscr{E}^{i}+(\stackrel{*}{\mathbf{P}} \times \mathbf{B})^{i}, \\
t^{[i j]}=0 \\
\rho \Sigma=t^{i j} \dot{x}_{i, j}+\stackrel{*}{\mathbf{P}} \cdot \mathscr{E}, \\
\frac{\partial \rho}{\partial t}+\left(\rho \dot{x}^{i}\right)_{, i}=0
\end{gathered}
$$

To this system of equations relating the motion $\tilde{\mathbf{x}}(\mathbf{X}, t)$, the polarization $\mathbf{P}$, the electromagnetic field $(\mathbf{E}, \mathbf{B})$, and the mass density $\rho$, one must add the electromagnetic equations (4.8)-(4.9).

In the simpler classical treatments of dielectrics, the electromotive intensity $\&$ is a function only of the polarization as in (4.10); however, in more intricate theories, the electromotive intensity may also depend on time derivatives of the polarization and on other variables as well, as for example, in Voigt's relations (4.11). A simple theory of elastic dielectrics emerges now if we assume that the electromotive intensity is a function $\mathscr{E}(\mathbf{F}, \mathbf{P})$ only of the polarization and the deformation gradient $F$, and that it is independent of the rates of change of these quantities. Then by reasoning similar to that followed in Section 3 in the review of ordinary elasticity theory, we see that for the system of equations (4.8)-(4.9), (4.2)-(4.5) not to be overdetermined, it is sufficient to require that the angular momentum equation (5.3) and the energy equation (5.4) be satisfied identically as a consequence of the constitutive relations for the internal energy $\Sigma$, the stress $t_{i}^{j}$, and the electromotive intensity $\mathscr{S}$. To see what restrictions such an assumption places on the constitutive relations 
for these quantities we introduce the material measures of polarization $\Pi^{A}$ defined in terms of the polarization $\mathbf{P}$ and the deformation gradient $\mathbf{F}$ by

$$
\Pi^{A} \equiv|(\mathbf{X} / \mathbf{x})|^{-1} X^{A}{ }_{, i} P^{i} .
$$

These measures of polarization satisfy the important identity

$$
\Pi^{A}=|(\mathbf{X} / \mathbf{x})|^{-1} X^{A},{ }_{i}^{*} P^{i},
$$

which is our reason for introducing them. This identity states that the transform of the convected time flux of the polarization by the motion is equal to the material derivative of the measures of polarization $\Pi^{A}$. Using this relation, we can write the energy equation (5.4) in the following form.

$$
\rho\left(\frac{\partial \bar{\Sigma}}{\partial x_{, A}^{i}} \dot{x}_{, A}^{i}+\frac{\partial \bar{\Sigma}}{\partial \Pi^{A}} \dot{\Pi}^{A}\right)=t_{i}^{j} \dot{x}_{, A}^{i} X^{A}{ }_{, j}+|(\mathbf{x} / \mathbf{X})|^{-1} x_{, A}^{i} \mathscr{E}_{i} \dot{\Pi}^{A}
$$

where we assume that the energy is expressed as a function $\bar{\Sigma}(\mathbf{F}, \mathbf{I})$ of the deformation gradient and material measures of polarization. Thus if the stress and electromotive intensity are independent of the rates of change of $\mathbf{F}$ and $\boldsymbol{\Pi}$, and if the energy equation is to be an identity, we must have

$$
\begin{aligned}
& \bar{t}_{i}{ }^{j}=\rho \frac{\partial \bar{\Sigma}}{\partial x_{, A}^{i}} x_{, A}^{j}, \\
& \overline{\mathscr{E}}_{i}=\rho_{\circ} \frac{\partial \bar{\Sigma}}{\partial \Pi^{A}} X_{, i}^{A} .
\end{aligned}
$$

These are the stress-deformation-field relations of a simple theory of elastic dielectrics valid, nevertheless, for finite deformations and for moving media. A generalization will be considered later. These relations fix the constitutive relations for the stress and electromotive intensity in terms of the internal energy function $\bar{\Sigma}(\mathbf{F}, \mathbf{M})$. It is still necessary to restrict the form of this function so that the stress as given by (5.9) will satisfy the equation of balance of angular momentum (5.3) identically. The stress (5.9) will be symmetric and satisfy (5.3) if and only if the energy is invariant under all rigid rotations when the polarization is rotated rigidly with the material. That is, the function $\hat{\Sigma}(\mathbf{F}, \mathbf{P})$ must satisfy the functional relation

$$
\hat{\Sigma}\left(R_{j}^{i} x^{j}, A, R_{j}^{i} P^{j}\right)=\hat{\Sigma}\left(x_{, A}^{i}, P^{i}\right)
$$

for all rotation matrices $\mathbf{R}$ and for each value of the deformation gradient and polarization. As was shown in [1], if the energy function satisfies (5.11), then it must be expressible as a function $\tilde{\Sigma}\left(E_{A B}, \Pi^{A}\right)$ of the six independent measures of finite strain $E_{A B}$ and the material measures of polarization $\Pi^{A}$. When the energy is so expressed, we then have

$$
\begin{aligned}
t^{i j}=t^{j i} & =\rho \frac{\partial \tilde{\Sigma}}{\partial E_{A B}} x_{, A}^{i} x^{j}{ }_{, B} \\
\mathscr{E}_{i} & =\rho_{\circ} \frac{\partial \tilde{\Sigma}}{\partial \Pi^{A}} X^{A}{ }_{, i} .
\end{aligned}
$$


In our earlier wor $k$ [1] we introduced an asymmetric stress tensor defined in terms of an energy function $\widetilde{\tilde{\Sigma}}(\mathbf{F}, \pi)$, where $\pi \equiv \mathbf{P} / \rho$ is the polarization per unit mass, by

$$
L_{t} t_{i}^{j} \equiv \rho \frac{\partial \widetilde{\tilde{\Sigma}}}{\partial x_{, A}^{i}} x^{j}, A
$$

This formula for the local stress ${ }_{L} t_{i}{ }_{i}$ is formally similar to equation (5.9) for the symmetric stress $t^{i j}$, but the difference in the independent variables of the energy function in the two formula is essential. By applying the chain rule of partial differentiation, we may immediately contrast the two stress tensors. First note that

Thus

$$
\begin{aligned}
\Pi^{A} & =|(\mathbf{x} / \mathbf{X})| X^{A}{ }_{, i} P^{l} \\
& =\rho|(\mathbf{x} / \mathbf{X})| X^{A}{ }_{i} \pi^{l} .
\end{aligned}
$$

where

$$
\begin{aligned}
t_{i}{ }^{j} & =\rho \frac{\partial \widetilde{\tilde{\Sigma}}}{\partial x_{, A}^{i}} x_{, A}^{j}+\rho \frac{\partial \tilde{\tilde{\Sigma}}}{\partial \pi^{k}} \frac{\partial \pi^{k}}{\partial x_{, A}^{i}} x_{, A}^{j} \\
& ={ }_{L} t_{i}^{j}+\rho \frac{\partial \tilde{\Sigma}^{\tilde{\Sigma}}}{\partial \pi^{i}} \rho_{0}^{-1} \Pi^{A} x^{j}{ }_{, A} \\
& ={ }_{L} t_{i}^{j}+|(\mathbf{x} / \mathbf{X})|^{-1} \frac{\partial \tilde{\tilde{\Sigma}}}{\partial \pi^{i}} \Pi^{A} x^{j}{ }_{, A} \\
& ={ }_{L} t_{i}{ }^{j}-{ }_{L} E_{i} P^{j},
\end{aligned}
$$

$$
-{ }_{L} E_{i} \equiv \frac{\partial \tilde{\tilde{\Sigma}}}{\partial \pi^{i}}
$$

is the local field introduced also in [1]. Since the stress tensor $t^{i j}$ is symmetric when the energy is invariant under rigid motions, it follows from (5.16) that the local stress ${ }_{\mathrm{L}} t_{j}^{j}$ is not symmetric unless the polarization is parallel to the local field.

Equation (5.13) can be written in the form

$$
\mathscr{E}_{i}+{ }_{\llcorner} E_{i}=0,
$$

and, when so viewed, suggests a balance of forces acting on the charged particles which make up the polarized material point of the medium. Hence we call it, the equation of molecular equilibrium.

A slightly more general theory of dielectrics which is able to account for the phenomena of optical activity and the Faraday effect replaces the simple equation of molecular equilibrium (5.18) by the following one:

$$
\mathscr{E}+{ }_{\llcorner} \mathbf{E}+\mathbf{G} \times \stackrel{*}{\mathbf{P}}=0,
$$

where $\mathbf{G}$ is a vector invariant function of the deformation gradient, the polarization, and the electromagnetic field. We call it the gyration vector. This term in the equation of molecular equilibrium is similar to the term $\mathbf{c}_{\alpha}^{-}(\dot{\mathbf{P}} \times \mathbf{B})$ in Voigt's equation (4.11). Since the vector $\mathbf{G} \times \stackrel{*}{\mathbf{P}}$ is always normal to $\stackrel{*}{\mathbf{P}}$, this term does not affect the balance of energy as can be seen most easily from (5.4). 
We close this section by collecting in one place the complete set of field equations, boundary conditions, and constitutive relations of the theory.

The field equations

$$
\begin{array}{ll}
\frac{\partial \mathbf{B}}{\partial t}+\operatorname{curl} \mathbf{E}=0, & \operatorname{div} \mathbf{B}=0, \\
\operatorname{curl} \mathbf{H}-\frac{\partial \mathbf{D}}{\partial t}=0, & \operatorname{div} \mathbf{D}=0,
\end{array}
$$

where

$$
\begin{gathered}
\mathbf{H} \equiv \mathbf{B} / \mu_{0}+\dot{\mathbf{x}} \times \mathbf{P}, \quad \mathbf{D} \equiv \epsilon_{\circ} \mathbf{E}+\mathbf{P} . \\
\left.\rho \ddot{x}_{i}=t_{i, j}^{j}-(\operatorname{div} \mathbf{P}) \mathscr{E}_{i}+\stackrel{*}{\mathbf{P}} \times \mathbf{B}\right)_{i}, \\
\mathscr{E}+{ }_{\llcorner} \mathbf{E}+\mathbf{G} \times \stackrel{*}{\mathbf{P}}=0 .
\end{gathered}
$$

The jump conditions

$$
\begin{gathered}
-V \llbracket \mathbf{B} \rrbracket+\mathbf{n} \times \llbracket \mathbf{E} \rrbracket=0, \quad \mathbf{n} \llbracket \mathbf{B} \rrbracket=0, \\
\mathbf{n} \times \llbracket \mathbf{H} \rrbracket+\llbracket \mathbf{D} \rrbracket=0, \quad \mathbf{n} \cdot \llbracket \mathbf{D} \rrbracket=0, \\
V \llbracket \rho \dot{x}_{i}+\epsilon_{0}(\mathbf{E} \times \mathbf{B})_{t} \rrbracket+n_{j} \llbracket t_{i}^{j}+m^{j} \rrbracket=0, \\
-V \llbracket \rho \rrbracket+\mathbf{n} \cdot \llbracket \rho \mathbf{x} \rrbracket=0 .
\end{gathered}
$$

The constitutive relations

$$
\begin{aligned}
& t^{i j}=\rho \frac{\partial \tilde{\Sigma}}{\partial E_{A B}} x_{, A}^{i} x^{j}, B, \\
& E_{i}=-\rho_{\circ} \frac{\partial \tilde{\Sigma}}{\partial \Pi^{A}} X^{A}, \\
& G^{i}=\sigma^{i}(\mathbf{F}, \mathbf{P}, \mathbf{E}, \mathbf{B}) .
\end{aligned}
$$

The jump conditions which follow from the integral equations of balance must hold at any surface of possible discontinuity in the fields. In these relations, $V$ is the speed of the discontinuity surface relative to the frame of reference and $\mathbf{n}$ is its unit normal. We use the conventional notation $\llbracket A \rrbracket$ for the difference $A^{+}-A^{-}$of the limiting values $A^{+}$and $A^{-}$ of the quantity $A$ as the surface of discontinuity is approached from either side. These jump conditions are derived from the integral equations of balance most easily by first writing them in a four dimensional form as explained in $[2, \S 287]$. In (5.21) we have introduced the symmetric electromagnetic stress tensor $m^{i j}$ defined by

$$
m^{i j} \equiv \epsilon_{\circ} E^{i} E^{j}+B^{i} B^{j} / \mu_{\circ}-\frac{1}{2}\left(\epsilon_{\circ} E^{2}+B^{2} / \mu_{0}\right) .
$$

Note that the divergence of this tensor is the Lorentz force:

$$
\operatorname{div} \mathbf{m}=Q \mathbf{E}+\mathbf{J} \times \mathbf{B}=-(\operatorname{div} \mathbf{P}) \mathbf{E}+(\stackrel{*}{\mathbf{P}}-\operatorname{div} \mathbf{P} \dot{\mathbf{x}}) \times \mathbf{B}
$$




\section{THE EQUATIONS FOR SMALL DEFORMATIONS AND WEAK FIELDS SUPERIMPOSED ON A FINITE DEFORMATION AND STRONG ELECTROMAGNETIC FIELD}

In many applications of the theory of elastic dielectrics, it is sufficient to linearize the equations about an initial equilibrium state of finite deformation, polarization, electric field, and magnetic flux density. When the initial state is free of stress, polarization, and electromagnetic field, the linearized system of equations we shall obtain from the equations of the non-linear theory reduce to the linear equations of the classical theory of piezoelectricity. But the general theory and the linearization technique we apply here yields more. By choosing the initial equilibrium state appropriately, one gets a coupled system of linear equations for the electromagnetic field, polarization, and small displacements about the initially deformed configuration which are suitable for the analysis of various optical phenomena in deformed and polarized dielectrics. The photoelastic effect is only one example of numerous phenomena which are predicted and correlated by the non-linear theory in this way.

To derive the linear equations for the small displacements and weak field in the general case, care must be taken to retain all relevant terms. Although there are alternative methods, we derive them as follows. Let

$$
\circ x^{i}, \circ \Pi^{A}, \circ E_{i}, \circ B^{i}
$$

denote any time independent solution of the non-linear equations for a given material. Let

$$
\begin{aligned}
& \tilde{x}^{i}(\mathbf{X}, t)={ }_{\circ} \tilde{x}^{i}(\mathbf{X})+\delta_{\mathbf{x}} x^{i}(\mathbf{X}, t), \\
& \Pi^{A}(\mathbf{X}, t)={ }_{0} \Pi^{A}(\mathbf{X})+\delta_{\mathbf{X}} \Pi^{A}(\mathbf{X}, t), \\
& E_{i}(\mathbf{x}, t)={ }_{0} E_{i}(\mathbf{X})+\delta_{\mathbf{x}} E_{i}(\mathbf{x}, t), \\
& B^{i}(\mathbf{x}, t)={ }_{0} B^{i}(\mathbf{x})+\delta_{x} B^{i}(\mathbf{x}, t),
\end{aligned}
$$

be any other solution, in general, time dependent. It is important to note that the independent variables in $\tilde{x}^{i}$ and $\Pi^{A}$ are material co-ordinates; whereas, the independent variables in $E_{i}$ and $B^{i}$ are spatial co-ordinates.

First consider the equation $(5.20)_{7}$ and, using the identity,

$$
\left[(\mathbf{x} / \mathbf{X})^{-1} x_{, A}^{i}\right]_{, i} \equiv 0
$$

transform it so that it reads

$$
\rho_{\circ} \ddot{x}_{i}=T_{i, A}^{A}-\operatorname{div} \Pi \mathscr{E}_{i}+e_{i j k} \dot{\Pi}^{A} B^{k} x^{j}, A,
$$

where

$$
\begin{aligned}
T^{A}{ }_{i} & \equiv \rho_{\circ} \frac{\partial \tilde{\Sigma}}{\partial E_{A B}} x_{i, B}, \\
& =\rho_{\circ} \frac{\partial \tilde{\Sigma}}{\partial E_{A B}} x^{k}{ }_{, B} g_{i k}, \\
& =|(\mathbf{x} / \mathbf{X})| X^{A}{ }_{, i} t_{i}{ }^{j},
\end{aligned}
$$

is the Piola-Kirchhoff stress tensor (cf. $[2, \S 210]$ ). 
Now each of the variations $\delta_{\mathbf{x}}$ of (6.2) commutes with material differentiation, i.e. with ( ) and ( ) $)_{A}$. Thus, computing the variation $\delta_{\mathbf{X}}$ of equation (6.3) we get

$$
\rho_{\circ} \frac{\ddot{\delta_{\mathbf{x}} x_{i}}}{}=\left(\delta_{\mathbf{x}} T_{i}^{\mathrm{A}}\right)_{, \mathbf{A}}-\operatorname{div}\left(\delta_{\mathbf{X}} \boldsymbol{\Pi}\right) \mathscr{E}_{i}-\operatorname{div} \boldsymbol{\Pi} \delta_{\mathbf{X}} \mathscr{E}_{i}+\mathrm{e}_{i j k} \frac{\dot{\delta_{\mathbf{X}} \Pi^{A}}}{B^{k} x^{j}, \boldsymbol{A}}
$$

where

$$
\delta_{\mathbf{X}} \mathscr{E}_{i}=\delta_{\mathbf{x}} \mathscr{E}_{i}+\mathscr{o}_{i, j} \delta_{\mathbf{X}} x^{j}
$$

But

$$
\left.\delta_{x} \mathscr{E}_{i}=\delta_{x} E_{i}+\frac{\dot{\left(\delta_{\mathbf{x}} \mathbf{x}\right.}}{\mathrm{o}} \mathbf{\mathrm { B }}\right)_{i},
$$

so that

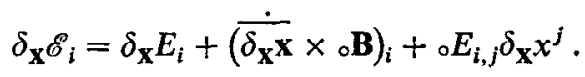

In (6.5) we have set div $\Pi=\Pi^{A}{ }_{, A}$. Next we compute $\delta_{\mathbf{X}} T_{i}^{A}$ using

$$
\delta_{\mathbf{X}} E_{A B}=\frac{1}{2} g_{m n}\left(x^{m}{ }_{, A} \delta_{\mathbf{X}} x^{n}{ }_{, B}+\delta_{\mathbf{X}} x^{m}{ }_{, A} x^{n},{ }_{B}\right)
$$

and find that

$$
\delta_{\mathbf{X}} T_{i}^{A}=\frac{\partial^{2} \tilde{\Sigma}}{\partial E_{A B} \partial E_{C D}} x_{i, B} \delta_{\mathbf{X}} E_{A B}+\frac{\partial^{2} \tilde{\Sigma}}{\partial E_{A B} \partial \Pi^{c}} x_{i, B} \delta \Pi^{c}+\frac{\partial \tilde{\Sigma}}{\partial E_{A B}} \delta_{\mathbf{X}} x_{i, B} .
$$

Multiplying equation (6.5) by ${ }_{\mathrm{o}}(\mathbf{x} / \mathbf{X} \mid$ we find that it can be put in the form

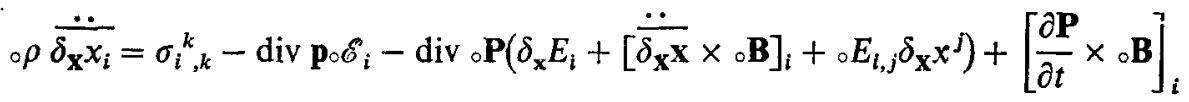

where

and we have put

$$
\begin{aligned}
& \sigma^{i k}=\operatorname{lo}_{0}(\mathbf{x} / \mathbf{X}) \mid{ }_{\circ} x_{, A}^{i} \delta_{\mathbf{X}} T^{i A} \\
& ={ }_{0} C^{i k j l}\left(\delta_{\mathbf{x}} x_{j}\right)_{l}+{ }_{o} S^{i k}{ }_{l}{ }^{l}+{ }_{o} t^{k l}\left(\delta_{\mathbf{x}} x^{i}\right)_{, l}
\end{aligned}
$$

$$
\begin{aligned}
& p^{i}=|\circ(\mathbf{x} / \mathbf{X})| \circ x_{, A}^{i} \delta_{\mathbf{X}} \Pi^{A},
\end{aligned}
$$

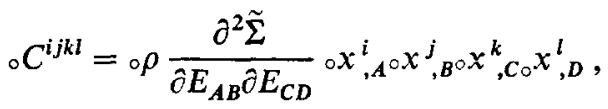

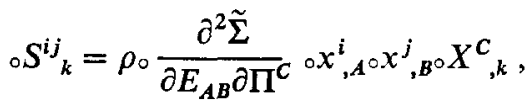

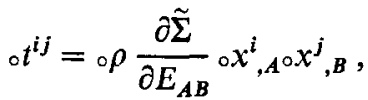

$$
\begin{aligned}
& { }_{\circ} \rho=\left.\right|_{\circ}(\mathbf{x} / \mathbf{X}) \mid \rho_{\circ} .
\end{aligned}
$$

We call $t_{0}{ }^{i j}$ the initial stress, ${ }_{0} C_{i j k l}$ the elastic moduli of the deformed material, and ${ }_{0} S^{i j}{ }_{k}$ the piezoelectric moduli of the deformed and polarized material. The quantity ${ }_{\mathrm{o}} \rho$ is the density of mass in the initially deformed configuration. Note that the increment of stress $\sigma^{\text {ik }}$ defined in (6.10) is, in general, not symmetric owing to the term linear in the initial stress. On the other hand, the moduli (6.12) and (6.13) have all the usual symmetry of the linear theory of piezoclectricity. 
It is possible to rewrite the term $\sigma_{, k}^{i k}$ in the equations of motion (6.9) in terms of a symetric increment of stress $\bar{\sigma}^{i k}$ given by

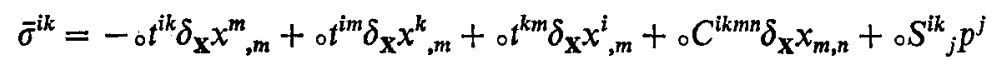

and which is related to $\sigma^{i k}$ through the identity

$$
\sigma_{, k}^{i k}=\bar{\sigma}_{, k}^{i k}-\mathrm{ot}^{i k}{ }_{, l} \delta_{\mathbf{X}} x_{, k}^{l} .
$$

Using (6.17), one can eliminate the term $\sigma^{i k}{ }_{, k}$ from the equations of motion so that, except for the electromagnetic terms on the right-hand side, we have the divergence of a symmetric increment of stress whenever the initial stress is homogeneous. We mention this possibility only because symmetric stress increments are viewed by some as having special significance. We attach no physical or mathematical significance to this possibility.

Next we linearize equation (5.20) using similar arguments and obtain

$$
\delta_{\mathbf{x}} E_{i}+\left(\dot{\overline{\delta_{\mathbf{x}} \mathbf{x}}} \times{ }_{\circ} \mathbf{B}\right)_{i}+{ }_{\circ} E_{i, j} \delta_{\mathbf{x}} x^{j}+{ }_{L} e_{i}+\left(\frac{\partial \mathbf{p}}{\partial t} \times \mathbf{G}\right)_{i}=0
$$

where ${ }_{L} e$ is the increment in the local field given by

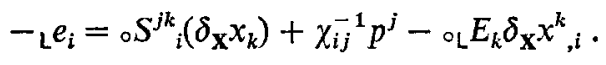

In (6.19), ${ }_{\mathrm{oL}} \mathbf{E}$ is the local field in the initial state of deformation and polarization, ${ }_{0} S^{j k}{ }_{i}$ are the piezoelectric moduli defined in (6.13) and $\chi_{i j}$ is the polarizability tensor of the deformed state defined by

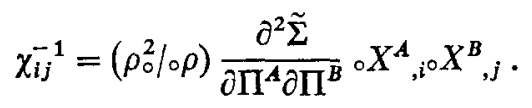

Next we linearize $(5.20)_{2}$ and $(5.20)_{3}$ using similar reasoning we obtain

$$
\begin{gathered}
\left(\epsilon_{\circ} \sqrt{ }(g) g^{i j} \delta_{\mathbf{x}} E_{j}\right)_{, i}=-\left(p^{i}+\delta_{\mathbf{x}} x^{k}{ }_{\circ} P_{, k}^{i}+\delta_{\mathbf{x}} x_{,{ }^{\circ}} P^{j}-\delta_{\mathbf{X}} x^{k}{ }_{, k}^{\circ} P^{i}\right)_{, i} \\
\operatorname{curl} \mu_{\circ}{ }^{-1} \delta_{\mathbf{x}} \mathbf{B}-\epsilon_{\circ} \frac{\partial \delta_{\mathbf{x}} \mathbf{E}}{\partial t}=\frac{\partial \mathbf{P}}{\partial t}+\operatorname{div}{ }_{\circ} \mathbf{P} \frac{\dot{P}}{\delta_{\mathbf{x}} \mathbf{x}}
\end{gathered}
$$

Finally, the remaining Maxwell equations are linearized without difficulty. We have

$$
\operatorname{curl} \delta_{\mathbf{x}} \mathbf{E}+\frac{\partial \delta_{\mathbf{x}} \mathbf{B}}{\partial t}=0, \quad \operatorname{div} \delta_{\mathbf{x}} \mathbf{B} .
$$

Now that the explicit but clumsy notations $\delta_{\mathbf{x}} \mathbf{E}, \delta_{\mathbf{x}} \mathbf{x}$, and $\delta_{\mathbf{x}} \mathbf{B}$ have served their purpose of keeping the distinction between the independent variables $\mathbf{X}$ and $\mathbf{X}$ closely drawn, we replace them by the more convenient notations

$$
\delta_{\mathbf{x}} \mathbf{E}=\mathbf{e}, \quad \delta_{\mathbf{x}} \mathbf{B}=\mathbf{b}, \quad \delta_{\mathbf{x}} \mathbf{x}=\mathbf{u} .
$$

Using these notations and collecting results we get for the complete system of linearized equations for the determination of $\mathbf{n}, \mathbf{p}, \mathbf{e}$, and $\mathbf{b}$. 


$$
\begin{gathered}
{ }_{\circ} \rho \ddot{u}_{i}=\sigma_{i, k}^{k}-\operatorname{div} \mathbf{p} \mathscr{E}_{i}-\operatorname{div}{ }_{\circ} \mathbf{P}\left(e_{i}+\left[\dot{\mathbf{u}} \times{ }_{\circ} \mathbf{B}\right]_{i}+{ }_{\circ} E_{i, j} u^{j}\right)+\left(\dot{\mathbf{p}} \times{ }_{\circ} \mathbf{B}\right)_{i} \\
e_{i}+\left[\dot{\mathbf{u}} \times{ }_{\circ} \mathbf{B}\right]_{i}+{ }_{\circ} E_{i, j} u^{j}+{ }_{L} e_{i}+[\dot{\mathbf{p}} \times \mathbf{G}]_{i}=0, \\
\epsilon_{\circ} \operatorname{div} \mathbf{e}=-\left(p^{i}+u_{\circ}{ }_{\circ} P^{i}{ }_{, k}+u_{, k}{ }^{i} P^{i}-u^{k}{ }_{, k^{\circ}} P^{i}\right)_{, i} \\
\operatorname{curl} \mathbf{b} / \mu_{\circ}-\epsilon_{\circ} \dot{\mathbf{e}}-\dot{\mathbf{p}}+\operatorname{div}{ }_{\circ} \mathbf{P} \mathbf{u}=0, \\
\operatorname{curl} \mathbf{e}+\mathbf{b}=0, \quad \operatorname{div} \mathbf{b}=0,
\end{gathered}
$$

where a superposed dot now stands for ordinary partial differentiation with respect to $t$. These field equations must be supplemented by the constitutive relations

$$
\begin{aligned}
\sigma^{i k} & ={ }_{\circ} C^{i k j l} u_{j, l}+{ }_{\circ} S^{i k}{ }_{l}^{l}+{ }_{\circ} t^{k l} u_{, l}^{i}, \\
-{ }_{L} e_{i} & ={ }_{\circ} S^{j k}{ }_{i} u_{j, k}+\chi_{i j}^{-1} p^{j}-{ }_{o L} E_{i} u^{k}{ }_{, k},
\end{aligned}
$$

where the coefficients ${ }_{0} C^{i j k l},{ }_{0} S^{i j}, \chi_{i j}^{-1}$, and ${ }_{0} t^{i j}$ are given by (6.12), (6.13), (6.14), and (6.20).

When the initial stress and the initial local field vanish, then the constitutive relations (6.29) and (6.30) reduce to Voigt's classical linear piezoelectric constitutive relations. Thus, (6.29) and (6.30) can be regarded as a generalization of the linear Voigt theory which takes into account the effects of initial stress and electric field or polarization. Again note that the stress $\sigma^{i k}$ is not symmetric unless the initial stress vanishes.

\section{THE CLASSICAL LINEAR THEORY OF PIEZOELECTRICITY}

Voigt's linear theory is such an important special case that we here summarize the linear system of equations which underlie it. These are obtained from the equations of the previous sections on assuming that the initial stress, the initial electric field, the initial polarization, and the gyration vector vanish. The equations of the previous section then simplify to the following set

$$
\begin{gathered}
\rho_{\circ} \ddot{u}_{i}=\sigma_{i, k}^{k}, \\
\mathbf{e}+{ }_{L} \mathbf{e}=0, \\
\operatorname{curl} \mathbf{b} / \mu_{\circ}-\epsilon_{\circ} \dot{\mathbf{e}}-\dot{\mathbf{p}}=0, \quad \operatorname{div}\left(\epsilon_{\circ} \mathbf{e}+\mathbf{p}\right)=0, \\
\operatorname{curl} \mathbf{e}+\mathbf{b}=0, \quad \operatorname{div} \mathbf{b}=0,
\end{gathered}
$$

where $\sigma^{i k}$ and $e_{i}$ are given by

$$
\begin{aligned}
\sigma^{i k} & ={ }_{\circ} C^{i k m n} u_{m, n}+{ }_{\circ} S^{i k}{ }_{j} p^{j}, \\
-{ }_{L} e_{i} & ={ }_{\circ} S^{m n}{ }_{i} u_{m, n}+{ }_{\circ} \chi_{i j}^{-1} p^{j} .
\end{aligned}
$$

Equations (7.1) and the constitutive relations (7.2) are the basic equations of the classical linear theory of piezoelectricity.

\section{THE FARADAY EFFECT AND MAGNETO-ELASTIC DRAGGING}

When a transparent dielectric medium which is normally isotropic is traversed by a plane polarized electromagnetic wave along the direction of a strong externally applied magnetic field, the plane of polarization of the wave is observed to rotate. This is the Faraday effect. The phenomenon does not occur in vacuum and, in most materials, the 
amount of rotation is proportional to the strength of the applied magnetic field, at least for sufficiently weak fields. The Faraday effect may also be described by saying that application of a magnetic field makes right and left circularly polarized light travel at slightly different speeds through the medium in the direction of the magnetic field. By magneto-elastic dragging we mean a new effect whereby the speed of a transverse sound wave traversing an elastic dielectric is depressed by application of a strong magnetic field in the direction of propagation of the wave. The purpose of this section is to demonstrate that the theory of dielectrics set forth above predicts these two phenomena.

For simplicity we consider only isotropic materials for which the moduli ${ }_{\circ} C^{i j k l},{ }_{0} S^{i j}{ }_{k}$, and ${ }^{i j}$ have the following special form in the undeformed and unpolarized state of the medium:

$$
\begin{aligned}
{ }_{\circ} C^{i j k l} & =\hat{\lambda} g^{i j} g^{k l}+\mu\left(g^{i k} g^{j l}+g^{i l} g^{k j}\right), \\
{ }_{0} \chi^{i j} & =\epsilon_{\circ} \chi g^{i j}, \\
{ }_{0} S^{i j}{ }_{k} & =0 .
\end{aligned}
$$

We shall assume that $\chi>0, \mu>0$, and $\lambda+2 \mu>0$. The constants $\lambda$ and $\mu$ are the Lamé constants for the material and the dimensionless parameter $\chi$ is the specific polarizability. The system of equations for a small displacement and weak field superimposed on such an initial state of an isotropic material then take the form

$$
\begin{gathered}
\rho_{\circ} \ddot{\mathbf{u}}=(\hat{\lambda}+\mu) \operatorname{grad} \operatorname{div} \mathbf{u}+\mu \nabla^{2} \mathbf{u}+\dot{\mathbf{p}} \times \circ \mathbf{B}, \\
\mathbf{e}+\mathbf{u} \times{ }_{\circ} \mathbf{B}-\left(1 / \epsilon_{\circ} \chi\right) \mathbf{p}+\mathbf{G} \times \dot{\mathbf{p}}=0, \\
\operatorname{curl} \mathbf{b} / \mu_{\circ}-\epsilon_{\circ} \dot{\mathbf{e}}-\dot{\mathbf{p}}=0, \quad \operatorname{div}\left(\epsilon_{\circ} \mathbf{e}+\mathbf{p}\right)=0, \\
\operatorname{curl} \mathbf{e}+\dot{\mathbf{b}}=0, \quad \operatorname{div} \mathbf{b}=0 .
\end{gathered}
$$

In an isotropic material, the gyration vector must have the form

$$
\mathbf{G}=\gamma\left({ }_{\circ} B^{2}\right)_{\circ} \mathbf{B}
$$

where $\gamma$ is a scalar valued function of ${ }_{0} B^{2}$, the square of the magnitude of the applied magnetic field.

To exhibit the Faraday effect and magnetoelastic dragging it is sufficient to examine solutions of this system of linear equations having the form of plane waves. For this purpose we set

$$
\begin{aligned}
& \mathbf{u}=\mathfrak{R} \overrightarrow{\mathbf{u}} \mathrm{e}^{i(k \mathbf{n} \cdot \boldsymbol{x}-\omega t)}, \quad \mathbf{p}=\mathfrak{R} \overline{\mathbf{p}} \mathrm{e}^{\mathrm{i}(k \mathbf{n} \cdot \boldsymbol{x}-\omega t)}, \\
& \mathbf{b}=\mathfrak{R} \mathbf{b} \mathrm{e}^{i(k \mathbf{n} \cdot x-\omega t)}, \quad \mathbf{e}=\mathfrak{R} \overline{\mathbf{e}} \mathrm{e}^{i(k \mathbf{n} \cdot \boldsymbol{x}-\omega t)},
\end{aligned}
$$

where $\mathbf{u}, \mathbf{p}, \overline{\mathbf{b}}$, and $\overline{\mathbf{e}}$ are complex amplitude vectors independent of $\mathbf{x}$ and $t, \omega>0$ is a real constant called the angular frequency of the wave, $k$ is the wave number, and $\mathbf{n}$ is a unit vector. $\mathfrak{R}$ denotes the real part of the quantity placed after it.

On substituting the assumed form of the solution (8.4) into the system of equations (8.2) we find that $k, \omega, \overline{\mathbf{u}}, \overline{\mathbf{p}}, \overline{\mathbf{b}}$, and $\overline{\mathbf{e}}$ must satisfy jointly the following system of homogeneous linear equations: 


$$
\begin{gathered}
\rho_{\circ} \omega^{2} \overline{\mathbf{u}}=(\lambda+\mu) k^{2} \mathbf{n} \cdot \overline{\mathbf{u}} \mathbf{n}+\mu k^{2} \mathbf{u}+i \omega \overline{\mathbf{p}} \times{ }_{\circ} \mathbf{B} \\
\overline{\mathbf{e}}-i \omega \overline{\mathbf{u}} \times{ }_{\circ} \mathbf{B}-\left(\epsilon_{\circ} \chi\right)^{-1} \overline{\mathbf{p}}-i \omega \gamma_{\circ} \mathbf{B} \times \overline{\mathbf{p}}=0 \\
\mu_{\circ}{ }^{-1} k \mathbf{n} \times \overline{\mathbf{b}}+\epsilon_{\circ} \omega \overline{\mathbf{e}}+\omega \overline{\mathbf{p}}=0, \\
k \mathbf{n} \times \overline{\mathbf{e}}-\omega \overline{\mathbf{b}}=0, \\
\mathbf{n} \cdot\left(\epsilon_{\circ} \overline{\mathbf{e}}+\overline{\mathbf{p}}\right)=0, \quad \mathbf{n} \cdot \overline{\mathbf{b}}=0 .
\end{gathered}
$$

When $\omega>0$, equations (8.9) are satisfied as a consequence of equations (8.7) and (8.8). The system (8.5)-(8.8) is a system of sixteen linear homogeneous equations in the sixteen unknowns $\bar{u}_{i}, \bar{e}_{i}, \bar{p}_{i}$, and $\bar{b}_{i}$. If the system has any non-trivial solution, the determinant of the coefficients must vanish. This condition imposes a single relation between the frequency $\omega$ and the wave number $k$. Rather than attempting to discuss the nature of the plane wave solution for a general direction of the impressed magnetic field, we shall consider only the special case when ${ }_{0} \mathbf{B}$ is parallel to the direction of propagation $\mathbf{n}$. Taking the scalar product of (8.7) and (8.8) by $\mathbf{n}$ then yields the two equations

$$
\mathbf{n} \cdot \overline{\mathbf{e}}=\mathbf{n} \cdot \overline{\mathbf{p}}=0
$$

and, when $\mathbf{n} \cdot \overline{\mathbf{p}}=0$, the equation (8.5) for $\mathbf{u}$ separates into the two equations

$$
\begin{gathered}
{\left[(\lambda+2 \mu) k^{2}-\rho_{\circ} \omega^{2}\right](\mathbf{n} \cdot \overline{\mathbf{u}})=0,} \\
\left(\mu k^{2}-\rho_{\circ} \omega^{2}\right)(\mathbf{n} \times \overline{\mathbf{u}})+i \omega_{\circ} B \overline{\mathbf{p}}=0 .
\end{gathered}
$$

From (8.11), we see that unless $(\mathbf{n} \cdot \overline{\mathbf{u}})=0$, the factor $\left[(\lambda+2 \mu) k^{2}-\rho_{\circ} \omega^{2}\right]$ must be zero. This condition fixes the speed $v=\omega / k$ of the longitudinal sound wave at the value,

$$
v=\sqrt{\left(\frac{\lambda+2 \mu}{\rho_{\circ}}\right),}
$$

which is independent of the applied magnetic field. When $\omega$ and $k$ are not related as in (8.13), we must have $\mathbf{n} \cdot \overline{\mathbf{u}}=0$, which means that $\mathbf{u}$ is transverse along with $\mathbf{e ,} \mathbf{p}$ and $\mathbf{b}$. To obtain the dispersion relation in this case, we eliminate $\bar{b}$ from (8.7) using (8.8) to get

$$
\overline{\mathbf{e}}=\frac{\beta^{2}}{\epsilon_{0}\left(1-\beta^{2}\right)} \overline{\mathbf{p}},
$$

where $\beta^{2}=v^{2} / c^{2}=\omega^{2} /\left(k^{2} c^{2}\right)$. We then eliminate $\overline{\mathbf{e}}$ from (8.6) using (8.14) to get

$$
\frac{1-\kappa \beta^{2}}{1-\beta^{2}} \overline{\mathbf{p}}+i \omega \chi \gamma_{\circ} B \epsilon_{\circ} \mathbf{n} \times \mathbf{p}-i \omega \chi \varepsilon_{\circ} \mathbf{n} \times \overline{\mathbf{u}}=0,
$$

where we have set $\kappa=1+\chi$. Taking the scalar product of (8.12) with $\overline{\mathbf{u}}$ yields $\overline{\mathbf{u}} \cdot \overline{\mathbf{p}}=0$, and taking the scalar product of (8.15) with $\mathbf{n} \times \overline{\mathbf{p}}$ yields

$$
i \omega \chi \gamma_{\circ} B \epsilon_{\circ}(\mathbf{n} \times \overline{\mathbf{p}}) \cdot(\mathbf{n} \times \overline{\mathbf{p}})-i \omega \chi \varepsilon_{0}(\mathbf{n} \times \overline{\mathbf{p}}) \cdot(\mathbf{n} \times \overline{\mathbf{u}})=0 .
$$

Now $(\mathbf{a} \times \mathbf{b}) \cdot(\mathbf{a} \times \mathbf{c})=\mathbf{a} \cdot \mathbf{a b} \cdot \mathbf{c}-\mathbf{a} \cdot \mathbf{b a} \cdot \mathbf{c}$ so that, using the results $\overline{\mathbf{u}} \cdot \overline{\mathbf{p}}=\overline{\mathbf{n}} \cdot \overline{\mathbf{p}}=$ $\overline{\mathbf{n}} \cdot \overline{\mathbf{p}}=\mathbf{0}$ already obtained for this case, we get

$$
i \omega \chi \gamma_{\circ} B \epsilon_{\circ}(\overline{\mathbf{p}} \cdot \overline{\mathbf{p}})=0 \text {. }
$$


Hence, unless $\omega \gamma \chi_{\circ} B=0$, we must have

$$
\overline{\mathbf{p}} \cdot \overline{\mathbf{p}}=0 .
$$

This requires that the real and imaginary parts of the complex amplitude $\overline{\mathbf{p}}$ be perpendicular and of equal length. The polarization wave is circularly polarized (cf. [7]) and

$$
\mathbf{n} \times \overline{\mathbf{p}}= \pm i \overline{\mathbf{p}}
$$

where the upper sign holds for the right and the lower sign for the left circularly polarized wave. Eliminating $\mathbf{n} \times \overline{\mathbf{p}}$ from (8.15) using (8.19) and $\mathbf{n} \times \overline{\mathbf{u}}$ using (8.12) we get

$$
\left[\frac{1-\kappa \beta^{2}}{1-\beta^{2}}+\frac{A_{1} \beta^{2}}{\beta^{2}-\beta_{s}^{2}} \pm A_{2}\right] \overline{\mathbf{p}}=0
$$

where we have put

$$
\begin{aligned}
v_{s}^{2} / c^{2} \equiv \beta_{s}^{2} & \equiv \frac{\mu}{\rho_{\circ} c^{2}}, \quad \kappa=1+\chi, \\
A_{1} & \equiv \frac{\epsilon_{\circ} \chi_{\circ} B^{2}}{\rho_{\diamond}}, \\
A_{2} & \equiv \chi \gamma_{\circ} B \omega .
\end{aligned}
$$

The dimensionless quantity $\beta_{s}^{2} \ll 1$ is the square of the ratio of the speed of transverse sound waves in the dielectric when $B=0$ to the speed of light in vacuum. For transverse sound waves to exist we see from (8.20) that we must have

$$
\frac{1-\kappa \beta^{2}}{1-\beta^{2}}+\frac{A_{1}}{\beta^{2}-\beta_{s}^{2}} \pm A_{2}=0 .
$$

This is the dispersion relation for transverse waves with a direction of propagation parallel to the applied magnetic field. The upper and lower signs on the dimensionless parameter $A_{2}$ in the dispersion relation refer to the right and left circularly polarized waves, respectively. Thus, unless $A_{2}=0$, we see that right and left circularly polarized waves will travel at different speeds. Thus $A_{2}$ is a measure of the Faraday effect. The dimensionless parameter $A_{1}$ will be very small in practice. For example, if the magnetic field is $1 \mathrm{~Wb} / \mathrm{m}^{2}=10^{4} \mathrm{G}$, the polarizability is 10 , and the mass density is $8 \times 10^{3} \mathrm{~kg} / \mathrm{m}^{3}$, then the parameter $A_{1}$ is approximately $10^{-9}$. The dimensionless parameter $A_{2}$ will also be very small. Retaining only first order terms in $\beta_{s}^{2}, A_{1}$, and $A_{2}$ in the solution of the dispersion equation (8.22), we find that the speed of the slow wave is given approximately by

$$
v^{2}=v_{s}^{2}\left[\frac{1 \pm A_{2} / \beta_{s}^{2}}{1+\left(A_{1} \pm A_{2}\right) / \kappa}\right] .
$$

The speed of the fast wave in the same approximation is given by

$$
v^{2}=c^{2}\left[\frac{1+A_{1} \mp A_{2}}{k+A_{1} \pm A_{2}}\right] .
$$

From (8.23) we see that the speeds of both the left and right-circularly polarized waves are depressed below their normal value by the presence of the coefficient $A_{1}$. It might be possible to detect this 'magneto-elastic dragging' by a suitable experimental arrangement. 
Other electro- and magneto-optical phenomena are predicted and correlated by the general system of equations of Section 6 . One has only to choose the initial electric and magnetic fields appropriately and to examine the solutions for electromagnetic waves in the material.

\section{ISOTROPIC DIELECTRICS AND THE PHOTOELASTIC EFFECT}

If the material is isotropic, then the energy function must satisfy the invariance condition

$$
\tilde{\Sigma}\left(E_{A B}, \bar{\Pi}^{A}\right)=\tilde{\Sigma}\left(E_{A B}, \Pi^{A}\right)
$$

where

$$
\bar{E}_{A B}=O^{M}{ }_{A} O^{N}{ }_{B} E_{M N}, \quad \bar{\Pi}^{A}=O_{M}{ }^{A} \Pi^{M},
$$

and $O_{B}^{A}$ is an arbitrary orthogonal transformation.

Then by arguments given in [1], it can be shown that any energy function satisfying (9.1) is expressible as a function of the following list of scalar invariants:

$$
\begin{aligned}
& J_{1}=E_{A}^{A}=\operatorname{tr} \mathbf{E}, \quad J_{2}=E^{A}{ }_{B} E^{B}{ }_{A}=\operatorname{tr} \mathbf{E}^{2}, \quad J_{3}=\operatorname{tr} \mathbf{E}^{3}, \\
& J_{4}=\Pi^{A} \Pi_{A} \cdot, \quad J_{5}=E_{A B} \Pi^{A} \Pi^{B}, \quad J_{6}=\left(E^{2}\right)_{A B} \Pi^{A} \Pi^{B} .
\end{aligned}
$$

When the initial state is a deformed state without initial polarization, electric field, or magnetic field, the equations for $\mathbf{e}, \mathbf{b}$, and $\mathbf{p}$ separate from the equation for $\mathbf{u}$. The equations for the determination of $\mathbf{e}, \mathbf{b}$, and $\mathbf{p}$ take the simple form

$$
\begin{gathered}
\operatorname{div}\left(\epsilon_{\circ} \mathbf{e}+\mathbf{p}\right)=0, \quad \operatorname{curl} \mu_{\circ}^{-1} \mathbf{b}-\frac{\partial}{\partial t}\left(\epsilon_{0} \mathbf{e}+\mathbf{p}\right)=\mathbf{0}, \\
\operatorname{curl} \mathbf{e}+\frac{\partial \mathbf{b}}{\partial t}=0, \quad \operatorname{div} \mathbf{b}=0, \\
\mathbf{e}+{ }_{\llcorner} \mathbf{e}=0,
\end{gathered}
$$

where, for isotropic materials, ${ }_{L} \mathbf{e}$ is given by

with $\chi_{i j}$ given by (6.20).

$$
-{ }_{L} e_{i}=\circ \chi_{i j}^{-1} p^{j}
$$

Expressing the internal energy as a function of the invariants (9.3) and working out the derivatives in (6.20) we find that, in an initially deformed isotropic dielectric with no electric or magnetic field impressed, the polarizability tensor has the form

$$
\circ \chi_{i j}=A \circ g_{i j}+A_{1} \circ c_{i j}+A_{2} \circ c_{i j}^{-1}
$$

where ${ }_{c} c^{i j}=x^{i}{ }_{,} x^{j}{ }^{A}$, and the coefficients $A_{0}, A_{1}$, and $A_{2}$ are certain functions of the strain invariants $J_{1}, J_{2}$, and $J_{3}$.

According to (9.6), the polarizability tensor, hence also the dielectric tensor, ${ }_{0} K_{j}{ }_{j}=$ $\delta_{j}^{i}+{ }_{o} \chi_{j}^{i}$, in the deformed dielectric have the same principal directions as the spatial strain tensor ${ }_{0} c_{j}{ }_{j}$. The initial stress tensor ${ }_{0} t_{j}^{i}$ also has this property.

Unless the strain tensor ${ }_{0} c_{j}^{i}$ is proportional to the unit tensor as in a uniform dilitation, the dielectric tensor $K_{j}^{i}$ will have unequal eigenvalues and the material will be birefringent. Since equations (9.4)-(9.5) are the customary basis for the analysis of electromagnetic wave propagation in the optical or linear approximation of weak fields, we see that the usual 
theory of photoelasticity is reproduced here as a special case of our general equations. The advantage gained by the general theory is the possibility now to examine the photoelastic effect in anisotropic materials and to correlate it to other properties of an elastic dielectric in a perfectly rational, deductive way without adding further hypotheses. For anisotropic materials, the invariance condition (9.1) must hold with the orthogonal group replaced by the appropriate symmetry group for the material under consideration.

\section{THE DRAGGING OF LIGHT BY A MOVING DIELECTRIC}

For the purposes of this section, it is sufficient that we consider a rigid, linear, isotropic dielectric for which the energy function has the special form

$$
\rho_{\circ} \Sigma=P^{2} ;\left(2 \epsilon_{\circ} \chi \sqrt{ } g\right), \quad \chi>0 .
$$

For such a material, the local field is given by

$$
-{ }_{\llcorner} \mathbf{E}=\mathbf{P} /(\epsilon \circ \chi) \text {. }
$$

We shall assume for simplicity that the gyration vector vanishes. The relevant equations for moving media are given in (5.5) and (5.6) where $\dot{\mathbf{x}}$ is the velocity field corresponding to a rigid motion.

Let the light wave be represented as a moving surface of discontinuity in $\mathbf{P}, \mathbf{E}$, and $\mathbf{B}$ (i.e. an electromagnetic shock front). Across such a surface of discontinuity, the jumps in $\mathbf{P}, \mathbf{E}$, and $\mathbf{B}$ must satisfy the system of equations (5.6). This yields a system of twelve homogeneous linear equations for the twelve components of the jumps in $\mathbf{P}, \mathbf{E}$, and $\mathbf{B}$. In order that the system have non-trivial solutions, it is necessary for the determinant of the matrix of coefficients of the system to vanish. This condition fixes the speed of propagation $V$ of the moving surface of discontinuity in terms of the other parameters of the problem. When the normal to the surface of discontinuity and the velocity of the medium are parallel, this condition has the simple form

$$
K(V / c)^{2}-2 \chi(\dot{x} / c)(V / c)+\chi(\dot{x} / c)^{2}-1=0,
$$

where $K=1+\chi$. If we let $N=K^{1 / 2}$ denote the index of refraction of the stationary medium we can write the solution of (10.3) in the form

$$
(v / c)=(1 / N) \pm \chi \dot{x} \dot{x} /(N c)+0\left(\dot{x}^{2} / c^{2}\right),
$$

where $O\left(\dot{x}^{2} / c^{2}\right)$ denotes terms of order $\dot{x}^{2} / c^{2}$ which may be neglected when the speed of the material is everywhere small compared with the speed of light in vacuum. The formula (10.3) agrees with the experimental data of Fizeau on the dragging of light by moving water (cf. [5, p. 110]). The formula was first given by Fresnel who derived it from a theory of the aether.

\section{A GENERALIZATION OF THE VOIGT THEORY AND ITS APPLICATION} TO A ROTATING RIGID DIELECTRIC

Consider a medium of the type treated by Voigt for which the polarization and its time derivatives are related to the electric field by an equation of the form (4.11). For simplicity, we shall assume that, when the medium is at rest,

$$
a \frac{\partial^{2} \mathbf{P}}{\hat{c} t^{2}}+(\epsilon \circ \chi)^{-1} \mathbf{P}=\mathbf{E} \text {. }
$$


The term in the second time derivative of $\mathbf{P}$ derives from the inertia of the charged particles making up the molecule. For simplicity, we neglect rotary effects which we have seen can be accounted for by a term like $\mathbf{G} \times \stackrel{*}{\mathbf{P}}$ in the equation of molecular equilibrium.

Now there are several generalizations of (11.1) to moving media which would reduce to (11.1) when the medium is stationary. One such generalization replaces the derivatives with respect to time by convected time derivatives and the electric field by the electromotive intensity to obtain

$$
a^{* *} \mathbf{P}+\left(\epsilon_{\circ} \chi\right)^{-1} \mathbf{P}=\mathbf{E}+\dot{\mathbf{x}} \times \mathbf{B} .
$$

This equation is invariant under arbitrary transformations to rotating co-ordinate systems. But if we think of the first term in equation (11.1) as arising from inertia, it would seem more appropriate to replace it by a $\ddot{\mathbf{P}}$ to obtain

$$
\mathbf{a} \ddot{\mathbf{P}}+(\epsilon \circ \chi)^{-1} \mathbf{P}=\mathbf{E}+\dot{\mathbf{x}} \times \mathbf{B}
$$

as the equation of molecular equilibrium in a moving medium whose stationary state is characterized by (11.1). The first term in (11.3) now gives rise to certain apparent forces in rotating co-ordinate systems. To see the effect of such inertial terms on a simple solution of our equations, we consider the experiment of Wilson [7] in which a hollow rotating cylinder of dielectric material is rotated in a magnetic field directed along its axis. The inside and outside surfaces of the cylinder are coated with a conducting material and connected through brushes with an electrometer of capacity $C$. When the magnetic field is turned on and the cylinder rotated, a voltage is developed between the brushes and the electrometer becomes charged.

Choose cylindrical co-ordinates and assume a solution for $\mathbf{E}, \mathbf{P}$, and $\mathbf{B}$ having the form

$$
\mathbf{E}=(\bar{E}, 0,0), \quad \mathbf{P}=(r \bar{P}, 0,0), \quad \mathbf{B}=(0,0, r \bar{B}) .
$$

The metric has components

$$
\left\|g_{l j}\right\|=\left\|\begin{array}{ccc}
1 & 0 & 0 \\
0 & r^{2} & 0 \\
0 & 0 & 1
\end{array}\right\| .
$$

The quantities $\bar{E}, \bar{P}$, and $\bar{B}$ represent the physical components of the electric field, polarization, and magnetic flux density, respectively.

In (4.8) and (4.9) we have written the relevant equations of electromagnetic theory in a form which applies to arbitrary curvilinear co-ordinate systems. For a rigidly rotating medium we find that

$$
\ddot{\mathbf{P}}=\left(-\omega^{2} r^{2} \bar{P}, 0,0\right) \text {. }
$$

Assuming a cylindrically symmetric solution of the form (11.4) where all quantities depend only on the radial co-ordinate $x^{1}=r$, and are independent of $x^{2}$ and $x^{3}$, equations (4.8), (4.9), and (11.1) reduce to

$$
\begin{gathered}
(\mathrm{d} / \mathrm{d} r)\left[r\left(\epsilon_{\circ} \bar{E}+\bar{P}\right)\right]=0, \\
(\mathrm{~d} / \mathrm{d} r)\left[\omega r \bar{P}-\mu_{\circ}{ }^{-1} \bar{B}\right]=0, \\
\left(1-a \omega^{2} \chi\right) \bar{P}=\epsilon_{\circ} \chi(E+\omega r \bar{B}) .
\end{gathered}
$$


We may write $(11.7)_{3}$ as follows:

where

$$
\vec{P}=\epsilon_{\circ} \chi^{\prime}(\bar{E}+\omega r \vec{B}),
$$

$$
\chi^{\prime}=\chi /\left(1-a \omega^{2} \chi\right)
$$

is the effective polarizability of the rotating medium.

The two differential equations of (11.7) integrate immediately to give

$$
\begin{gathered}
r\left(\epsilon_{\circ} \bar{E}+\bar{P}\right)=k_{1}, \\
\omega r \bar{P}-\mu_{\circ}^{-1} \bar{B}=k_{2},
\end{gathered}
$$

where $k_{1}$ and $k_{2}$ are constants to be evaluated by the boundary data of the experiment.

To evaluate the constants $k_{1}$ and $k_{2}$ we assume first that the net charge on the inner and outer surfaces of the rotating dielectric cylinder is zero so that the electric field exterior to the cylinder vanishes. Then considering a small pill-box divided by the exterior surface of the cylinder we conclude that

$$
2 \pi \llbracket r_{2} \epsilon_{\circ} \bar{E}\left(r_{2}\right)+\bar{P}\left(r_{2}\right) \rrbracket=2 \pi k_{1}=\Omega,
$$

where $\Omega$ is the free charge per unit length of the cylinder deposited there by the electrometer. If $L$ denotes the length of the cylinder, then the total charge of one sign which has flowed from the electrometer is given by $Q=\Omega L$ from which we conclude that

$$
k_{1}=C V /(2 \pi L) \text {, }
$$

where $V$ is the voltage registered by the electrometer and $C$ is its capacity.

To evaluate the constant $k_{2}$ we note that, for an infinitely long cylinder, the magnetic field caused by the rotating charges within it or on its surface vanishes at points exterior to the cylinder. Let ${ }_{0} B$ then denote the applied field and consider a small circuit divided by the exterior surface of the rotating cylinder. There will be a certain free current through such a circuit arising from the free charge deposited there by the electrometer. The charge per unit time per unit length of the surface passing through the circuit is $\Omega \omega /(2 \pi)$. Thus applying the integral condition (4.2) to such a circuit and correcting for the presence of the free current, we get the boundary condition

$$
\llbracket \omega r P-\mu_{0}^{-1} B \rrbracket=\Omega \omega /(2 \pi),
$$

where the quantity on the left denotes the jump or difference between the limiting values of the enclosed quantity as the surface of the cylinder is approached from the outside and inside, respectively. But from (11.10) and (11.13) we conclude that

$$
k_{2}=-\mu_{\circ}^{-1}{ }_{o} B+C V \omega /(2 \pi L) \text {. }
$$

We now solve the three equations $(11.8),(11.10)_{1}$ and $(11.10)_{2}$ for $E$ and get

$$
\epsilon_{\circ}\left(1+\frac{\chi^{\prime}}{1-r^{2} \omega^{2} / c^{2}}\right) \vec{E}=k_{1} / r+\frac{\chi^{\prime} \omega r k_{2}}{c^{2}\left(1-r^{2} \omega^{2} / c^{2}\right)} .
$$

But $r^{2} \omega^{2}$ is the speed of the medium and if we neglect the terms $r^{2} \omega^{2} / c^{2}$ compared to 1 , we can easily integrate equation (11.14) between the limits $r_{1}$ and $r_{2}$, the inner and outer radii of the cylinder. In this way we get the condition

$$
-\epsilon_{\circ} K^{\prime} V=k_{1} \log \left(r_{2} / r_{1}\right)+\chi^{\prime} \omega k_{2}\left(r_{2}^{2}-r_{1}^{2}\right) /\left(2 c^{2}\right) \text {. }
$$


Substituting now for $k_{1}$ and $k_{2}$ from (11.12) and (11.14) we get the convenient formula for the voltage

$$
V=\frac{\chi^{\prime} f A_{\circ} B}{K^{\prime}(1+C / C)},
$$

where we have put $f=\omega /(2 \pi)$, and

$$
C^{\prime}=2 \pi L K^{\prime} / \log \left(r_{2} / r_{1}\right), \quad A=\pi\left(r_{2}^{2}-r_{1}^{2}\right) .
$$

The quantity $f$ in (11.16) is the rate of rotation measured in revolutions per unit time, $C^{\prime}$ is the capacity of the rotating cylinder, and $A$ is the area of its cross section.

The formula (11.16) with $\chi^{\prime}$ and $K^{\prime}$ replaced by $\chi$ and $K$ agrees with the formula given by Cullwick [8]. If the inertial term in (11.3) is zero or negligible, the voltage given by (11.16) is a linear function of the rate of rotation. If a careful experiment should reveal a departure from a linear relation between the voltage and the rate of rotation, it might be explained by the inertial contributions to the modified formula (11.16).

\section{COMPARISONS WITH OTHER THEORIES}

Born and Huang [9, Chap. VI] assume that the free energy $\Phi$ of a polarizable, deformable, stationary elastic medium is a function

$$
\Phi=\hat{\Phi}(\mathbf{F}, \mathbf{E})
$$

of the deformation gradient $\mathbf{F}$ and the electric field $\mathbf{E}$. They conclude that if the free energy is invariant under rigid rotations of the material when the electric field is rotated rigidly with the material that $\Phi$ reduces to a function

$$
\Phi=\tilde{\Phi}\left(E_{A B}, \bar{E}_{A}\right)
$$

of the measures of finite strain $E_{A B}$ and the quantities $\bar{E}_{A}$ defined by

$$
\bar{E}_{A} \equiv E_{i} X_{, A}{ }_{,}
$$

To this point we agree with their hypotheses and conclusions. However, they arrive somehow at an energy equation which states in effect that

where,

$$
\left(\rho / \rho_{\circ}\right) \dot{\Phi}=S^{i j} d_{i j}-E^{[i} P^{j j} \omega_{i j},
$$

$$
d_{i j}=\dot{x}_{(i, j)}, \quad \omega_{i j}=\dot{x}_{[i, j]} .
$$

They call the quantity $S^{i j}$ the stress. We recognize $d_{i j}$ as the rate of deformation tensor and $\omega_{i j}$ as the vorticity. Except for the electromagnetic term in (12.4), this equation would agree with the energy equation of finite elasticity theory. After laying down equation (12.4) without prior discussion, the authors then consider a motion or 'variation' for which they impose the constraint

$$
\dot{E}_{i}=0,
$$

and require that, for all such variations, (12.4) be satisfied identically.

Now when (12.6) holds, we have

$$
\dot{\vec{E}}_{A}=\dot{x}_{, A}^{i} E_{i}=\dot{x}_{i, j} x^{j}{ }_{, A} E^{i} .
$$


Thus we may match the coefficients in the following equation

to get

$$
\left(\rho / \rho_{\circ}\right)\left(\frac{\partial \tilde{\Phi}}{\partial E_{A B}} x_{, A}^{i} x_{, B}^{j} \mathrm{~d}_{i j}+\frac{\partial \tilde{\Phi}}{\partial \bar{E}_{A}} x^{i}{ }_{, A} E^{i} \dot{x}_{i, j}\right)=S^{i j} \mathrm{~d}_{i j}-E^{[i} P^{i]} \omega_{i j}
$$

$$
S^{i j}=\left(\rho / \rho_{\circ}\right)\left(\frac{\partial \tilde{\Phi}}{\partial E_{A B}} x_{, A}^{i} x_{, B}^{j}+\frac{\partial \tilde{\Phi}}{\partial \bar{E}_{A}} x_{, A}^{(j} E^{i)}\right)
$$

and

$$
-E^{[i} p^{j]}=\left(\rho / \rho_{\circ}\right) \frac{\partial \Phi}{\partial \bar{E}_{A}} x^{[j}, E^{i]},
$$

which are the equations (37.25) and (37.26) of Born and Huang [9] in our notation.

By an independent argument, Born and Huang then set

$$
P^{i}=-\left(\rho / \rho_{\circ}\right) \frac{\partial \tilde{\Phi}^{\tilde{E}}}{\partial \bar{E}_{A}} x_{A}^{i},
$$

which agrees with our result in that $\mathbf{P}$, in the static case, is a rotationally invariant function of the electric field and deformation gradient. As they claim, (12.11) is consistent with (12.10); on the other hand, it cannot be concluded from it.

It is difficult to criticize the formula (12.9) which they give for the stress since nowhere in their book are the equations given which this stress is supposed to satisfy. One is left to infer that, even in the fully general case where non-linear terms in the electromagnetic field are to be retained, the equations of motion for the medium are supposed to be

$$
S^{i j}{ }_{j}=\rho \ddot{x}^{i},
$$

with $S^{i j}$ given by (12.9). If (12.12) represents a correct inference, then we may say definitely that the theory of Born and Huang and the theory developed in the preceding chapters are inequivalent.

The whole difficulty in the Born and Huang treatment seems to stem from the energy equation (12.4). This equation appears inconsistent to us by the following reasoning. The free energy $\Phi$ is supposed to be invariant under rigid motions. This is assumed by Born and Huang and we agree that the free energy should have this property. But consider an anisotropic dielectric for which $\mathbf{P}$ is not parallel to $\mathbf{E}$, at least for some directions of $\mathbf{E}$. Let such a material be used as the insulator of a simple flat plate condensor. Now on rotating the condensor rigidly so that the electric field between its plates rotates with it, the polarization as given by (12.11) also rotates rigidly with the material. But if the anisotropic material is cut in such a manner that $\mathbf{P}$ is not parallel to $\mathbf{E}$ the energy equation of Born and Huang (12.4) leads to a contradiction for, in such a motion, no deformation occurs so that $d_{i j}=0$; the material is rotating so that $\omega_{i j} \neq 0$; and (12.4) says that $\dot{\boldsymbol{\Phi}} \neq 0$. But, by assumption, $\dot{\mathbf{\phi}}=0$ in a rigid motion. It may be, of course, that we have misinterpreted the Born and Huang equations. On the other hand, if our interpretation has been correct, the forgoing remarks would seem to prove inconsistency of their theory.

We consider next the non-linear stress-strain-field relations derived by Mason [10]. In [10] there appears an energy function $\tilde{U}$ such that

$$
T^{i j}=\frac{\partial \tilde{U}}{\partial \tilde{E}_{i j}}, \quad E_{i}=\frac{\partial \tilde{U}}{\partial D^{i} / \epsilon_{\circ}},
$$


where $\tilde{\tilde{E}}_{i j} \equiv \frac{1}{2}\left(u_{i, j}+u_{j, i}\right)$ is the symmetric part of the displacement gradient. Now as we have seen, if the energy is invariant under rigid motions, then it is impossible to express it as a function only of the symmetric part of the displacement gradient and one of the vectors $\mathbf{E}, \mathbf{D}$, or $\mathbf{P}$, in an exact non-linear treatment of large deformations and/or large rotations. Only in the linear approximation and in the absence of initial stress will the stress tensor $t^{i j}$, or $t^{i j}$ depend only on the deformation through the quantities $\widetilde{E}_{i j}$ only. However, here again as in the case of Born and Huang, it proves difficult to appraise the formulae for the stress $T^{i j}$ given in Mason's paper. The equations of motion determined by $T^{i j}$ are nowhere recorded. Again one is left to infer by analogy with the linear theory of Voigt that they are intended to be of the form

$$
T^{i j}{ }_{j}=\rho \ddot{x}^{i} .
$$

If this be a correct inference, then Mason's theory and ours agree only in the linear approximation and in the absence of initial stress.

Now the equations of motion

$$
t^{i j}{ }_{, j}=\rho \ddot{x}^{i}
$$

can be written in the form

$$
T^{A B}{ }_{, B} x_{i, A}^{i}+T^{A B} x_{, A B}^{i}=\ddot{x}^{i},
$$

where

$$
T^{A B}=\rho_{\circ} \frac{\partial \widetilde{U}}{\partial E_{A B}} .
$$

Thus if we were to identify the energy function $\tilde{U}$ with $\rho_{0} \Sigma$ and replace the infinitesimal strain measures $\tilde{\tilde{E}}_{i j}$ by the finite strain measures $E_{A B}$ and $D^{i}$ by

$$
\bar{D}^{A}=|(\mathbf{x} / \mathbf{X})| X^{A}{ }_{, i} D^{i},
$$

the formulae (12.13) given by Mason would be consistent with the invariance of the energy function under rigid motions. Also, with these reinterpretations of the dependent and independent variables, the remainder of Mason's paper dealing with definitions of the photoelastic constants etc. remain valid in the non-linear range.

\section{ACKNOWLEDGEMENTS}

I especially wish to thank Prof. J. L. Ericksen for the many discussions we have had concerning all aspects of this work. I wish also to express my appreciation for the assistance of Prof. A. C. Eringen and Mr. Neal Jordon who carefully checked the results of Section 8 and affirmed their consistency with the more extensive research on this aspect of the problem by Dunkin and Eringen [11].

\section{REFERENCES}

[1] R. A. ToupIN, J. Rat. Mech. Anal. 5, 849-916 (1956).

[2] C. A. Truesdell and R. A. ToupIn, The Classical Field Theories, Vol. III/1, Handbush der physik. Springer, Berlin (1960).

[3] H. Mimkowski, Math. Annalen 68, 472-525 (1910).

[4] A. Einstein and J. Laub, Ann. d. Phys. 26, 532-540 (1908). 
[5] E. T. WhitTAKer, A History of the Theories of Aether and Electricity, Vol. 1, The Classical Theories, Nelson, London (1951).

[6] W. Voigt, Ann. der Phys. 67, 345-365 (1899).

[7] H. A. Wilson, Phil. Trans. Roy. Soc. A, 204, 121 (1905).

[8] E, G. Cullwick, Electromagnetism and Relativity, Longmans, Green and Co., London (1957).

[9] M. BoRn and K. HuAng, Dynamical Theory of Crystal Lattices, Clarendon Press, Oxford (1954).

[10] W. P. Mason, Bell Syst. Tech.J. 29, 161-188 (1950).

[11] J. W. Dunkin and A. C. ERINGEn, Technical Report No. 18, Office of Naval Research, Contract Nonr-1100(02), (1961).

Résumé-On développe une théorie électromagnetique pour des milieux diélectriques en mouvement et susceptibles de déformations élastiques finies. On derive les equations d'un champ faible en superposition d'un champ fort, ainsi qu'un état arbitraire de déformation finie. On montre comment certains effets électro- et magnéto-optiques, ainsi que le ralentissement d'une onde sonore par un champ magnetique important peuvent être expliqués et illustrés dans un milieu diélectrique.

Zusammenfassung-Eine Theorie des elektromagnetischen Feldes in beweglichen elastischen Nichtleitern unter grossen Verformungen wird entwickelt. Die Gleichungen für ein schwaches Feld überlagert auf ein starkes Feld und einen beliebigen Zustand grosser Verformung, werden abgeleitet. Es wird gezeigt wie bestimmte elektro- und magneto-optische Effekte und die Verzögerung einer Schallwelle durch ein starkes magnetisches Feld in einen Nichtleiter erklärt und in Beziehung gesetzt werden können.

Sommario-È qui sviluppata una tcoria clettro-magnetica per dei dielettici in moto e suscettibili a deformazioni elastiche limitate. Si deducono le equazioni per un campo debole superimposto a un campo forte, come pure uno stato arbitrario di deformazione limitata. È mostrato come certi effetti elet ro e magneticoottici et come pure il rallentamento di un'onda sonora da un forte campo magnetico importante possano essere spiegati e correlati in un dielettrico.

Аннотация - Излагается теория әлектромагнитного поля в движущем, деформировнном эластическом диэлектрике. Выводятся уравнения слабого поля перекрывающего сильное поле, а также произвольное положение деформации.

Показывается что некоторые электро - и матнито-оптические аффекты и вамедление звуковой волны магнитным полем можно объяснить, и соотношение между ними можно установить. 